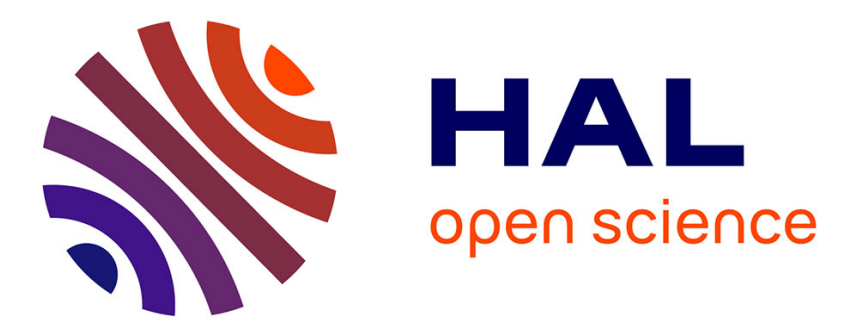

\title{
Discontinuous Galerkin Method for Frictional Interface Dynamics
}

\author{
Timothy Truster, Arif Masud
}

\section{To cite this version:}

Timothy Truster, Arif Masud. Discontinuous Galerkin Method for Frictional Interface Dynamics. Journal of Engineering Mechanics - ASCE, 2016, 142 (11), pp.04016084. 10.1061/(ASCE)EM.19437889.0001142 . hal-02437875

\section{HAL Id: hal-02437875 \\ https://hal.science/hal-02437875}

Submitted on 10 Nov 2021

HAL is a multi-disciplinary open access archive for the deposit and dissemination of scientific research documents, whether they are published or not. The documents may come from teaching and research institutions in France or abroad, or from public or private research centers.
L'archive ouverte pluridisciplinaire HAL, est destinée au dépôt et à la diffusion de documents scientifiques de niveau recherche, publiés ou non, émanant des établissements d'enseignement et de recherche français ou étrangers, des laboratoires publics ou privés.

\section{다)(1) $(5$}

Distributed under a Creative Commons Attribution - NonCommercial| 4.0 International 


\title{
Discontinuous Galerkin Method for Frictional Interface Dynamics
}

\author{
Timothy J. Truster ${ }^{1}$ and Arif Masud ${ }^{2}$
}

\begin{abstract}
A stabilized discontinuous Galerkin (DG) formulation is presented for transient small deformation contact problems involving friction with application to the modeling of bolted lap joints. The method is an extension of derivations from the quasi-static context, whereby the numerical flux terms acting at the contact interface are consistently derived using variational multiscale concepts. This transient primal formulation naturally accommodates nonconforming meshes and stratified materials such as geological faults. Also, the numerical flux terms involving the stress field at the contacting interface provide a natural mechanism for embedding friction models. Numerical results for nonsmooth transient problems confirm that the DG interface approach does not introduce artificial features into the physical response.
\end{abstract}

Keywords: Discontinuous Galerkin method; Elastodynamics; Interface; Stabilized methods; Friction.

\section{Introduction}

The characterization of dynamic response of contacting surfaces plays an important role in the behavior of civil engineering structures. Prominent examples include the sliding at faults during seismic activity, bolted connections in steel structures, and connections within bridges of high-speed rail systems. Often, the microscale compliance and damping characteristics at the interface serve as the main source for energy dissipation and vibrational resistance in engineered systems. At the relevant scales for continuum mechanics, these characteristics of the surface can be incorporated either through explicit representation of the surface roughness (Anciaux and Molinari 2010; Ladeveze et al. 2002) or through the interaction of nominally flat surfaces with statistically equivalent properties (Truster et al. 2013). In either case, the numerical treatment of the contact conditions is of utmost importance, particularly for capturing transient response.

Classical finite-element techniques for contact traditionally involve node-to-node penalty formulations, node-to-surface projections, or mortar methods to treat the discrete contact conditions; the latter have made significant progress in removing mesh biasing and solving finite strain problems consistently (Laursen et al. 2012; McDevitt and Laursen 2000; Temizer 2013). Another class of mixed formulations that have demonstrated efficient performance for contact problems are the dual mortar methods (Popp and Wall 2014; Sitzmann et al. 2015). The alternative to mixed methods is the primal formulation adopting ideas from the Nitsche method (Masud et al. 2012; Wriggers and Zavarise 2007) in which the displacement field is the only unknown. Extension of these primal formulations to embedded interface problems have also been applied to quasi-static frictional sliding (Annavarapu et al. 2014) and

\footnotetext{
${ }^{1}$ Assistant Professor, Dept. of Civil and Environmental Engineering, Univ. of Tennessee, Knoxville, TN 37921 (corresponding author). E-mail: ttruster@utk.edu

${ }^{2}$ Professor of Mechanics and Structures, Dept. of Civil and Environmental Engineering, Univ. of Illinois at Urbana-Champaign, Urbana, IL 61801.
}

explicit dynamics (Annavarapu et al. 2012b). For elastostatics problems, the performance of primal methods for problems with discontinuous material properties and nonconforming or embedded meshes (Annavarapu et al. 2012a; Truster and Masud 2014) has been linked to the definition of the numerical interface flux as a weighted average of the stresses across the interface. In the transient context, these interface flux terms may have an even greater effect because errors introduced at one time step will propagate during the remainder of the simulation. Thus, a proper definition for these flux terms in the presence of nonconforming meshes and nonlinearities due to friction is expected to be critical to maintaining stability within the numerical formulation.

In this work, a stabilized discontinuous Galerkin (DG) interface method is presented for transient contact analysis with friction. The developments are cast within the general framework of Truster and Masud (2014), which employs concepts from the variational multiscale (VMS) method (Hughes 1995) to consistently derive expressions for the numerical fluxes according to fine-scale models at the contact interface. The fine-scale modeling technique in this framework is coupled with an implicit time-integration scheme to address the dynamic response of domains containing interfaces. The ensuing numerical flux terms involve a weighted average of the interface stresses and serve to weakly impose displacement continuity and traction equilibrium. In the context of modeling contacting surfaces (Masud et al. 2012), the numerical flux terms are segregated into normal and tangential components and subsequently embed constitutive models for friction. The Coulomb friction model will be employed within the present work; extension to physics-based friction models follows along similar lines as pursued in Truster et al. (2013).

In the following sections, the DG formulation is presented first for fully bonded interfaces for transient problems and then is extended to contact and friction. Numerical results for impact test cases are presented with particular emphasis on isolating artificial effects, if any, that are induced by the DG interfaces on the computed dynamics.

\section{Stabilized Discontinuous Interface Method for Elastodynamics}

The present derivations mirror those of Truster and Masud (2014) for the quasi-static interface problem, while emphasis is placed on 


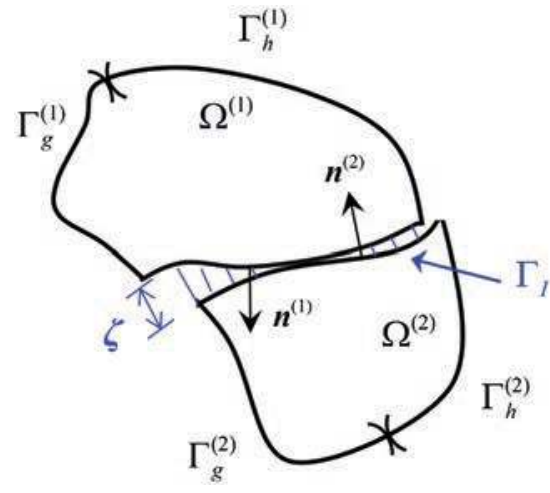

Fig. 1. (Color) Domain $\Omega$ split into two regions by interface $\Gamma_{I}$ with imposed discontinuity $\zeta$

assumptions relevant to the dynamic context. Consider an open, bounded domain $\Omega \subset \mathbb{R}^{n_{\mathrm{sd}}}$ and a time interval of interest $\square=] 0, T\left[\right.$, where $n_{\text {sd }}$ is the number of spatial dimensions. The domain $\Omega$ is split into two regions $\Omega^{(1)}$ and $\Omega^{(2)}$ by an interface $\Gamma_{I}$ as shown in Fig. 1. Along the interface $\Gamma_{I}$, the displacement field $\mathbf{u}: \Omega \times] 0, T\left[\rightarrow \mathbb{R}^{n_{\mathrm{sd}}}\right.$ has a prescribed discontinuity given by the field $\left.\zeta: \Gamma_{I} \times\right] 0, T\left[\rightarrow \mathbb{R}^{n_{\mathrm{sd}}}\right.$. Presently, $\zeta$ may be viewed as a rigid link or spacer between the regions; subsequently, it will be given the physical connotation of the penetration or slip at the interface. Under these conditions, the following elastodynamics initial-boundary value problem is posed within each region $(\alpha)$ for $\alpha=1,2$, and along $\Gamma_{I}$ :

$$
\begin{gathered}
\left.-\rho^{(\alpha)} \ddot{\mathbf{u}}^{(\alpha)}+\operatorname{div} \boldsymbol{\sigma}^{(\alpha)}+\mathbf{b}^{(\alpha)}=0 \quad \text { in } \Omega^{(\alpha)} \times\right] 0, T[ \\
\left.\mathbf{u}^{(\alpha)}=\mathbf{g}^{(\alpha)}(\mathbf{x}, t) \quad \text { on } \Gamma_{g}^{(\alpha)} \times\right] 0, T[ \\
\left.\boldsymbol{\sigma}^{(\alpha)} \cdot \mathbf{n}^{(\alpha)}=\mathbf{h}^{(\alpha)}(\mathbf{x}, t) \quad \text { on } \Gamma_{h}^{(\alpha)} \times\right] 0, T[ \\
\mathbf{u}^{(\alpha)}(\mathbf{x}, 0)=\mathbf{u}_{0}^{(\alpha)}(\mathbf{x}) \quad \text { in } \Omega^{(\alpha)} \\
\dot{\mathbf{u}}^{(\alpha)}(\mathbf{x}, 0)=\dot{\mathbf{u}}_{0}^{(\alpha)}(\mathbf{x}) \quad \text { in } \Omega^{(\alpha)} \\
\mathbf{u}^{(1)}-\mathbf{u}^{(2)} \equiv \llbracket \mathbf{u} \rrbracket=\zeta(\mathbf{x}, t) \quad \text { on } \Gamma_{I} \\
\boldsymbol{\sigma}^{(1)} \cdot \mathbf{n}^{(1)}=\lambda(\mathbf{x}, t)=-\boldsymbol{\sigma}^{(2)} \cdot \mathbf{n}^{(2)} \quad \text { on } \Gamma_{I}
\end{gathered}
$$

In the following developments, the superscript $(\alpha)$ will be suppressed when the specified field has counterparts in both regions $\Omega^{(\alpha)}$. The balance of linear momentum in Eq. (1) relates the divergence of the Cauchy stress $\boldsymbol{\sigma}$ to the body force $\mathbf{b}$ and the acceleration field $\ddot{\mathbf{u}}$. The prescribed displacement $\mathbf{g}$ is assigned on the Dirichlet boundary $\Gamma_{g}$ through Eq. (2), and the prescribed traction $\mathbf{h}$ is assigned on the Neumann boundary $\Gamma_{h}$ through Eq. (3), where $\Gamma_{g}^{(\alpha)} \cap \Gamma_{h}^{(\alpha)}=\emptyset$ and the region boundary $\Gamma^{(\alpha)}=\Gamma_{g}^{(\alpha)} \cup \Gamma_{h}^{(\alpha)} \cup \Gamma_{I}$. Also, $\mathbf{n}^{(\alpha)}$ is the unit outward normal on $\Gamma^{(\alpha)}$. Initial conditions in Eqs. (4) and (5) are ascribed to both the displacement $\mathbf{u}$ and velocity $\dot{\mathbf{u}}$ fields at time $t=0$. The displacement jump across the interface $\llbracket \mathbf{u} \rrbracket$ is set equal to the prescribed discontinuity $\zeta$ along $\Gamma_{I}$ via the constraint in Eq. (6). Finally, this constraint is enforced through the Lagrange multiplier field $\left.\lambda: \Gamma_{I} \times\right] 0, T\left[\rightarrow \mathbb{R}^{n_{\mathrm{sd}}}\right.$, which has the connotation of the traction field at the interface according to the equilibrium condition in Eq. (7).

To fix ideas, each region is considered as a homogeneous isotropic material possessing a mass density $\rho$, bulk modulus $K$, and shear modulus $G$; generalizations to anisotropic (Truster et al. 2015a) and inelastic (Truster 2015) materials are straightforward. The Cauchy stress is expressed as $\boldsymbol{\sigma}=K \operatorname{tr}(\boldsymbol{\varepsilon}) \mathbf{I}+$ $2 G[\boldsymbol{\varepsilon}-1 / 3 \operatorname{tr}(\boldsymbol{\varepsilon}) \mathbf{I}] \equiv \mathbf{C}: \boldsymbol{\varepsilon}$, where $\mathbf{I}$ is the second-order identity tensor, $\operatorname{tr}(\cdot)$ is the trace operator, and $\varepsilon$ is the small strain tensor. The small strain tensor is obtained through the symmetric gradient operator acting on the displacement field $\boldsymbol{\varepsilon} \equiv \boldsymbol{\varepsilon}(\mathbf{u})=$ $1 / 2\left[\nabla \mathbf{u}+(\nabla \mathbf{u})^{T}\right]$, where $\nabla(\cdot)$ and $(\cdot)^{T}$ denote the gradient and transpose operators, respectively.

A numerical approximation is now sought for the primary unknown fields $\{\mathbf{u}, \dot{\mathbf{u}}, \ddot{\mathbf{u}}, \boldsymbol{\lambda}\}$ solving Eqs. (1)-(7) as driven by the prescribed fields $\left\{\mathbf{b}, \mathbf{g}, \mathbf{h}, \mathbf{u}_{o}, \dot{\mathbf{u}}_{o}, \boldsymbol{\zeta}\right\}$. Herein, discretization will be applied first along the temporal axis and then along the spatial axes. Let the time interval $\square$ be discretized into a series of finite time intervals, with a generic interval denoted by times $\left[t_{n}, t_{n+1}\right]$ and the time step $\Delta t=t_{n+1}-t_{n}$. The Newmark- $\beta$ method is invoked to express the velocity $\dot{\mathbf{u}}_{n+1}$ and acceleration $\ddot{\mathbf{u}}_{n+1}$ at time $t_{n+1}$ in terms of the displacement $\mathbf{u}_{n+1}$ and known values $\left\{\mathbf{u}_{n}, \dot{\mathbf{u}}_{n}, \ddot{\mathbf{u}}_{n}\right\}$ at time $t_{n}$

$$
\begin{gathered}
\ddot{\mathbf{u}}_{n+1}=\frac{1}{\beta \Delta t^{2}} \mathbf{u}_{n+1}+\left(1-\frac{1}{2 \beta}\right) \ddot{\mathbf{u}}_{n}-\frac{1}{\beta \Delta t} \dot{\mathbf{u}}_{n} \\
-\frac{1}{\beta \Delta t^{2}} \mathbf{u}_{n} \equiv \frac{1}{\beta \Delta t^{2}} \mathbf{u}_{n+1}+\tilde{\mathbf{a}}_{n+1} \\
\dot{\mathbf{u}}_{n+1}=\frac{\gamma}{\beta \Delta t} \mathbf{u}_{n+1}+\left(1-\frac{\gamma}{\beta}\right) \dot{\mathbf{u}}_{n}+\gamma \Delta t\left(1-\frac{1}{2 \beta}\right) \ddot{\mathbf{u}}_{n} \\
-\frac{\gamma}{\beta \Delta t} \mathbf{u}_{n} \equiv \frac{\gamma}{\beta \Delta t} \mathbf{u}_{n+1}+\tilde{\mathbf{v}}_{n+1}
\end{gathered}
$$

where $\beta$ and $\gamma=$ parameters controlling temporal stability and order of accuracy; and $\left\{\tilde{\mathbf{v}}_{n+1}, \tilde{\mathbf{a}}_{n+1}\right\}=$ predictors defined to simplify the resulting expressions. Emphasis in the numerical simulations will be given to the familiar average acceleration method $\{\beta, \gamma\}=$ $\{1 / 4,1 / 2\}$.

Applying Eqs. (8)-(9) to Eqs. (1)-(7) leads to a sequence of boundary value problems written in strong form. The corresponding weak form is obtained by weakly enforcing Eqs. (1), (3), (6), and (7), which is stated as follows: Find $\left\{\mathbf{u}_{n+1}^{(1)}, \mathbf{u}_{n+1}^{(2)}, \lambda\right\} \in \mathcal{S}^{(1)} \times$ $\mathcal{S}^{(2)} \times \mathcal{Q}$ such that for all $\left\{\mathbf{w}^{(1)}, \mathbf{w}^{(2)}, \boldsymbol{\mu}\right\} \in \mathcal{V}^{(1)} \times \mathcal{V}^{(2)} \times \mathcal{Q}$

$$
\begin{gathered}
\sum_{\alpha=1}^{2} \int_{\Omega^{(\alpha)}} \mathbf{w}^{(\alpha)} \cdot \frac{\rho^{(\alpha)}}{\beta \Delta t^{2}} \mathbf{u}_{n+1}^{(\alpha)} \mathrm{d} \Omega+\sum_{\alpha=1}^{2} \int_{\Omega^{(\alpha)}} \boldsymbol{\varepsilon}\left(\mathbf{w}^{(\alpha)}\right): \mathbf{C}: \boldsymbol{\varepsilon}\left(\mathbf{u}_{n+1}^{(\alpha)}\right) \mathrm{d} \Omega \\
-\int_{\Gamma_{I}} \boldsymbol{\lambda}_{n+1} \cdot \llbracket \mathbf{w} \rrbracket \mathrm{d} \Gamma=\sum_{\alpha=1}^{2} \int_{\Omega^{(\alpha)}} \mathbf{w}^{(\alpha)} \cdot\left(\mathbf{b}_{n+1}^{(\alpha)}-\rho^{(\alpha)} \tilde{\mathbf{a}}_{n+1}^{(\alpha)}\right) \mathrm{d} \Omega \\
+\sum_{\alpha=1}^{2} \int_{\Gamma_{h}^{(\alpha)}} \mathbf{w}^{(\alpha)} \cdot \mathbf{h}_{n+1}^{(\alpha)} \mathrm{d} \Gamma \\
-\int_{\Gamma_{I}} \boldsymbol{\mu} \cdot\left(\llbracket \mathbf{u}_{n+1} \rrbracket-\boldsymbol{\zeta}_{n+1}\right) \mathrm{d} \Gamma=0
\end{gathered}
$$

where the appropriate function spaces are specified as

$$
\mathcal{S}^{(\alpha)}=\left\{\mathbf{u}^{(\alpha)}\left|\mathbf{u}^{(\alpha)} \in\left[H^{1}\left(\Omega^{(\alpha)}\right)\right]^{n_{\mathrm{sd}}}, \quad \mathbf{u}^{(\alpha)}(\mathbf{x})\right|_{\Gamma_{g}^{(\alpha)}}=\mathbf{g}^{(\alpha)}\left(\mathbf{x}, t_{n+1}\right)\right\}
$$




$$
\begin{gathered}
\mathcal{V}^{(\alpha)}=\left\{\mathbf{w}^{(\alpha)}\left|\mathbf{w}^{(\alpha)} \in\left[H_{0}^{1}\left(\Omega^{(\alpha)}\right)\right]^{n_{\mathrm{sd}}}, \quad \mathbf{w}^{(\alpha)}(\mathbf{x})\right|_{\Gamma_{g}^{(\alpha)}}=0\right\} \\
\mathcal{Q}=\left\{\boldsymbol{\lambda} \mid \boldsymbol{\lambda} \in\left[H^{-(1 / 2)}\left(\Gamma_{I}\right)\right]^{n_{\mathrm{sd}}}\right\}
\end{gathered}
$$

and $H^{1}\left(\Omega^{(\alpha)}\right)$ and $H^{-(1 / 2)}\left(\Gamma_{I}\right)$ are standard Sobolev spaces [see Barbosa and Hughes (1991) and Truster and Masud (2014) and references therein]. The next step is to convert the mixed interface formulation in Eqs. (10)-(11) into a primal formulation expressed in terms of the displacement field $\mathbf{u}_{n+1}^{(\alpha)}$ alone by condensing out the Lagrange multiplier field $\lambda_{n+1}$. This is achieved by applying a multiscale decomposition to separate $\mathbf{u}_{n+1}^{(\alpha)}$ into coarse scales $\overline{\mathbf{u}}_{n+1}^{(\alpha)}$ and fine scales $\mathbf{u}_{n+1}^{\prime(\alpha)}$ in the vicinity of $\Gamma_{I}$, according to the VMS framework presented in Truster and Masud (2014)

$$
\mathbf{u}_{n+1}^{(\alpha)}=\overline{\mathbf{u}}_{n+1}^{(\alpha)}+\mathbf{u}_{n+1}^{\prime(\alpha)}, \quad \mathbf{w}^{(\alpha)}=\overline{\mathbf{w}}^{(\alpha)}+\mathbf{w}^{\prime(\alpha)}
$$

As is typical for VMS formulations (Hughes 1995), herein the coarse scales will be associated with the finite-element displacement field, while the fine scales can be viewed as the error field filtered out by the given mesh. The modeling of numerical fine scales that are otherwise unaccounted for in the standard Galerkin method leads to solid mechanics formulations exhibiting enhanced stability (Cervera et al. 2003; Masud and Truster 2013; Masud et al. 2011; Masud and Xia 2006; Scovazzi et al. 2015). The key steps are subsequently provided for the derivation of the primal formulation using the multiscale approach while highlighting the distinguishing assumptions and terms compared to Truster and Masud (2014). First, the multiscale decomposition in Eq. (15) is substituted into the weak form in Eq. (10), and the terms corresponding to the fine-scale problem in each region $\Omega^{(\alpha)}$ associated with the fine-scale weighting terms $\mathbf{w}^{\prime(\alpha)}$ are as follows:

$$
\begin{aligned}
\int_{\Omega^{(\alpha)}} & \mathbf{w}^{\prime(\alpha)} \cdot \frac{\rho^{(\alpha)}}{\beta \Delta t^{2}} \mathbf{u}_{n+1}^{\prime(\alpha)} \mathrm{d} \Omega+\int_{\Omega^{(\alpha)}} \boldsymbol{\varepsilon}\left(\mathbf{w}^{\prime(\alpha)}\right): \mathbf{C}: \boldsymbol{\varepsilon}\left(\boldsymbol{u}_{n+1}^{\prime(\alpha)}\right) \mathrm{d} \Omega \\
= & +\int_{\Gamma_{I}} \mathbf{w}^{\prime(\alpha)} \cdot\left[(-1)^{\alpha-1} \boldsymbol{\lambda}_{n+1}-\mathbf{C}: \boldsymbol{\varepsilon}\left(\overline{\mathbf{u}}_{n+1}^{(\alpha)}\right) \cdot \mathbf{n}^{(\alpha)}\right] \mathrm{d} \Gamma \\
& +\int_{\Omega^{(\alpha)}} \mathbf{w}^{\prime(\alpha)} \cdot\left[\mathbf{b}^{(\alpha)}-\rho^{(\alpha)} \overline{\tilde{\mathbf{a}}}^{(\alpha)}-\frac{\rho^{(\alpha)}}{\beta \Delta t^{2}} \overline{\mathbf{u}}_{n+1}^{(\alpha)}+\operatorname{div} \boldsymbol{\sigma}\left(\overline{\mathbf{u}}_{n+1}^{(\alpha)}\right)\right] \mathrm{d} \Omega \\
& +\int_{\Gamma_{h}^{(\alpha)}} \mathbf{w}^{\prime(\alpha)} \cdot\left[\mathbf{h}_{n+1}^{(\alpha)}-\boldsymbol{\sigma}\left(\overline{\mathbf{u}}_{n+1}^{(\alpha)}\right) \cdot \mathbf{n}^{(\alpha)}\right] \mathrm{d} \Gamma
\end{aligned}
$$

where the terms involving the coarse-scale fields have been taken to the right-hand side and integration by parts has been applied. Because the fine-scale problem in Eq. (16) is infinite dimensional, modeling assumptions are applied from Truster and Masud (2014) in order to solve it analytically. First, the fine scales are taken to be nonzero only within the layer of elements adjoining the interface, which causes the Neumann term in Eq. (16) to vanish. Within each element $\Omega_{e}^{(\alpha)}$ adjacent to $\Gamma_{I}, \mathbf{u}_{n+1}^{\prime(\alpha)}$ is represented using an edge bubble functions $\mathbf{b}_{s}^{(\alpha)}$ that is supported over a sector of the element $\omega_{s}^{(\alpha)} \subseteq \Omega_{e}^{(\alpha)}$ and vanishes on all edges except the one edge intersecting the interface $\gamma_{s}=\partial \Omega_{e}^{(\alpha)} \cap \Gamma_{I}$. Although the coarse-scale discretization may be nonconforming along $\Gamma_{I}$, a set of segments $\left\{\gamma_{s}\right\}_{s=1}^{n_{\text {seg }}}$ can still be defined that covers $\Gamma_{I}$ and is conforming with respect to the sectors $\omega_{s}^{(\alpha)}$ on either side. This fine-scale representation enables Eq. (16) to be posed over the series of segments $\gamma_{s}$ and sectors $\omega_{s}^{(\alpha)}$ surrounding $\Gamma_{I}$. The specific definitions of $\gamma_{s}$ and $\omega_{s}^{(\alpha)}$ provided in Truster and Masud (2014) are adopted; the reader is referred to this reference for further details. Lastly, due to the significance of the interface in these developments, the interface traction residual is assumed to dominate over the contribution of the volumetric residual term in Eq. (16), leaving a single term on the right-hand side.

Substituting these assumptions into the fine-scale problem in Eq. (16) yields a segmentwise expression for $\mathbf{u}_{n+1}^{\prime(\alpha)}$ in terms of a consistently derived stabilization tensor $\boldsymbol{\tau}_{s}^{(\alpha)}$ accounting for the element geometry and material parameters

$$
\begin{gathered}
\left.\mathbf{u}_{n+1}^{\prime(\alpha)}\right|_{\gamma_{s}}=\boldsymbol{\tau}_{s}^{(\alpha)}\left[(-1)^{\alpha-1} \boldsymbol{\lambda}_{n+1}-\mathbf{C}: \boldsymbol{\varepsilon}\left(\overline{\mathbf{u}}_{n+1}^{(\alpha)}\right) \cdot \mathbf{n}^{(\alpha)}\right] \\
\boldsymbol{\tau}_{s}^{(\alpha)}=\left[\operatorname{meas}\left(\gamma_{s}\right)\right]^{-1}\left[\int_{\omega_{s}^{(\alpha)}} \boldsymbol{\varepsilon}\left(\mathbf{b}_{s}^{(\alpha)}\right): \mathbf{C}: \boldsymbol{\varepsilon}\left(\mathbf{b}_{s}^{(\alpha)}\right) \mathrm{d} \Omega\right]^{-1}\left[\int_{\gamma_{s}} b_{s}^{(\alpha)} \mathrm{d} \Gamma\right]^{2}
\end{gathered}
$$

where $\left[\operatorname{meas}\left(\gamma_{s}\right)\right]=$ surface area (arc length) of segment $\gamma_{s}$ for three (two)-dimensional problems. Eqs. (17) and (18) match exactly those derived in Truster and Masud (2014) because of the addition modeling assumption to drop the mass-type term from the fine scale on the left-hand side of Eq. (16). This assumption is consistent with the present treatment of the fine scales as piecewise constant in time within the temporally discrete weak form in Eqs. (10) to (11); also, Eq. (18) for $\boldsymbol{\tau}_{s}^{(\alpha)}$ provided excellent numerical performance in the tests in this paper. Herein, the simple polynomial bubble functions provided in Truster et al. (2015b) and Truster and Masud (2014) are chosen to represent the fine-scale bubbles $b_{s}^{(\alpha)}$ within Eq. (18).

Embedding the localized fine scales in Eq. (17) into the coarsescale problem associated with Eqs. (10) and (11) yields a stabilized mixed weak form for the coarse scales $\overline{\mathbf{u}}_{n+1}^{(\alpha)}$ and $\lambda_{n+1}$

$$
\begin{aligned}
& \sum_{\alpha=1}^{2} \int_{\Omega^{(\alpha)}} \overline{\mathbf{w}}^{(\alpha)} \cdot \frac{\rho^{(\alpha)}}{\beta \Delta t^{2}} \overline{\mathbf{u}}_{n+1}^{(\alpha)} \mathrm{d} \Omega+\sum_{\alpha=1}^{2} \int_{\Omega^{(\alpha)}} \boldsymbol{\varepsilon}\left(\overline{\mathbf{w}}^{(\alpha)}\right): \mathbf{C}: \boldsymbol{\varepsilon}\left(\overline{\mathbf{u}}_{n+1}^{(\alpha)}\right) \mathrm{d} \Omega \\
& \quad-\int_{\Gamma_{I}} \boldsymbol{\lambda}_{n+1} \cdot\left(\overline{\mathbf{w}}^{(1)}-\overline{\mathbf{w}}^{(2)}\right) \mathrm{d} \Gamma \\
& \quad+\sum_{\alpha=1}^{2} \int_{\Gamma_{I}}\left[\mathbf{C}: \boldsymbol{\varepsilon}\left(\overline{\mathbf{w}}^{(\alpha)}\right) \cdot \mathbf{n}^{(\alpha)}\right] \\
& \quad \cdot \boldsymbol{\tau}_{s}^{(\alpha)}\left[(-1)^{\alpha-1} \boldsymbol{\lambda}_{n+1}-\mathbf{C}: \boldsymbol{\varepsilon}\left(\overline{\mathbf{u}}_{n+1}^{(\alpha)}\right) \cdot \mathbf{n}^{(\alpha)}\right] \mathrm{d} \Gamma \\
& =\sum_{\alpha=1}^{2} \int_{\Omega^{(\alpha)}} \overline{\mathbf{w}}^{(\alpha)} \cdot\left(\mathbf{b}_{n+1}^{(\alpha)}-\rho^{(\alpha)} \overline{\mathbf{a}}_{n+1}^{(\alpha)}\right) \mathrm{d} \Omega+\sum_{\alpha=1}^{2} \int_{\Gamma_{h}^{(\alpha)}} \overline{\mathbf{w}}^{(\alpha)} \cdot \mathbf{h}_{n+1}^{(\alpha)} \mathrm{d} \Gamma \\
& -\int_{\Gamma_{I}} \boldsymbol{\mu} \cdot\left[\left(\overline{\mathbf{u}}_{n+1}^{(1)}-\overline{\mathbf{u}}_{n+1}^{(2)}\right)-\boldsymbol{\zeta}\right] \mathrm{d} \Gamma \\
& -\int_{\Gamma_{I}} \boldsymbol{\mu} \cdot\left[-\boldsymbol{\tau}_{s}^{(1)}\left[\mathbf{C}: \boldsymbol{\varepsilon}\left(\overline{\mathbf{u}}_{n+1}^{(1)}\right) \cdot \mathbf{n}^{(1)}\right]+\boldsymbol{\tau}_{s}^{(2)}\left[\mathbf{C}: \boldsymbol{\varepsilon}\left(\overline{\mathbf{u}}_{n+1}^{(2)}\right) \cdot \mathbf{n}^{(2)}\right]\right] \mathrm{d} \Gamma \\
& -\int_{\Gamma_{I}} \boldsymbol{\mu} \cdot\left[\boldsymbol{\tau}_{s}^{(1)}+\boldsymbol{\tau}_{s}^{(2)}\right] \cdot \boldsymbol{\lambda}_{n+1} \mathrm{~d} \Gamma=0
\end{aligned}
$$

As discussed in Truster and Masud (2014), the inherent stability of Eqs. (19) and (20) enables the choice of the discrete multiplier space $\mathcal{Q}^{h}=\left[L^{2}\left(\Gamma_{I}\right)\right]^{n_{\text {sd }}} \subset \mathcal{Q}$, which enables the analytical solution of the continuity Eq. (20) as follows:

$$
\left.\boldsymbol{\lambda}_{n+1}\right|_{\gamma_{s}}=\left.\left\{\mathbf{C}: \boldsymbol{\varepsilon}\left(\overline{\mathbf{u}}_{n+1}\right) \cdot \mathbf{n}\right\}\right|_{\gamma_{s}}-\left.\boldsymbol{\tau}_{s}\left(\llbracket \overline{\mathbf{u}}_{n+1} \rrbracket-\boldsymbol{\zeta}_{n+1}\right)\right|_{\gamma_{s}}
$$




$$
\begin{aligned}
& \{\boldsymbol{\sigma} \cdot \mathbf{n}\} \equiv \boldsymbol{\delta}_{s}^{(1)}\left[\boldsymbol{\sigma}^{(1)} \cdot \mathbf{n}^{(1)}\right]-\boldsymbol{\delta}_{s}^{(2)}\left[\boldsymbol{\sigma}^{(2)} \cdot \mathbf{n}^{(2)}\right] \\
& \boldsymbol{\tau}_{s}=\left(\boldsymbol{\tau}_{s}^{(1)}+\boldsymbol{\tau}_{s}^{(2)}\right)^{-1}, \quad \boldsymbol{\delta}_{s}^{(\alpha)}=\boldsymbol{\tau}_{s} \cdot \boldsymbol{\tau}_{s}^{(\alpha)}
\end{aligned}
$$

The distinguishing feature of Eq. (21) is that $\boldsymbol{\lambda}_{n+1}$ is a function of the prescribed discontinuity $\zeta_{n+1}$ through the stability tensor $\boldsymbol{\tau}_{s}$ scaling the constraint condition in Eq. (6); otherwise, the results of Truster and Masud (2014) remain unchanged. The weighting coefficients $\boldsymbol{\delta}_{s}^{(\alpha)}$ derived through the stability tensors $\boldsymbol{\tau}_{s}^{(\alpha)}$ provide a definition for the numerical interface traction in Eq. (22), which is well suited toward regions with material property mismatch such as fibrous composite materials as well as stratum within geological formations (Truster and Masud 2013, 2014).

Embedding the analytical expression Eq. (21) into Eq. (19) and combining terms leads to the stabilized primal formulation through the condensation of $\lambda_{n+1}$

$$
\begin{aligned}
\sum_{\alpha=1}^{2} & \int_{\Omega^{(\alpha)}} \mathbf{w}^{(\alpha)} \cdot \frac{\rho^{(\alpha)}}{\beta \Delta t^{2}} \mathbf{u}_{n+1}^{(\alpha)} \mathrm{d} \Omega+\sum_{\alpha=1}^{2} \int_{\Omega^{(\alpha)}} \boldsymbol{\varepsilon}\left(\mathbf{w}^{(\alpha)}\right): \mathbf{C}: \boldsymbol{\varepsilon}\left(\mathbf{u}_{n+1}^{(\alpha)}\right) \mathrm{d} \Omega \\
& +\int_{\Gamma_{I}} \llbracket \mathbf{w} \rrbracket \cdot \boldsymbol{\tau}_{s} \cdot\left(\llbracket \mathbf{u}_{n+1} \rrbracket-\zeta\right) \mathrm{d} \Gamma-\int_{\Gamma_{I}}[\mathbf{C}: \boldsymbol{\varepsilon}(\mathbf{w}) \cdot \mathbf{n}] \\
& \cdot\left(\llbracket \mathbf{u}_{n+1} \rrbracket-\boldsymbol{\zeta}\right) \mathrm{d} \Gamma \\
& -\int_{\Gamma_{I}} \llbracket \mathbf{w} \rrbracket \cdot\left[\mathbf{C}: \boldsymbol{\varepsilon}\left(\mathbf{u}_{n+1}\right) \cdot \mathbf{n}\right] \mathrm{d} \Gamma-\int_{\Gamma_{I}} \llbracket \mathbf{C}: \boldsymbol{\varepsilon}(\mathbf{w}) \cdot \mathbf{n} \rrbracket \cdot \boldsymbol{\delta}_{s} \\
& \cdot \llbracket \mathbf{C}: \boldsymbol{\varepsilon}\left(\mathbf{u}_{n+1}\right) \cdot \mathbf{n} \rrbracket \mathrm{d} \Gamma \\
= & \sum_{\alpha=1}^{2} \int_{\Omega^{(\alpha)}} \mathbf{w}^{(\alpha)} \cdot\left(\mathbf{b}_{n+1}^{(\alpha)}-\rho^{(\alpha)} \tilde{\mathbf{a}}_{n+1}^{(\alpha)}\right) \mathrm{d} \Omega+\sum_{\alpha=1}^{2} \int_{\Gamma_{h}^{(\alpha)}} \mathbf{w}^{(\alpha)} \cdot \mathbf{h}_{n+1}^{(\alpha)} \mathrm{d} \Gamma
\end{aligned}
$$

where the additional traction jump term $\llbracket \boldsymbol{\sigma} \cdot \mathbf{n} \rrbracket \equiv \boldsymbol{\sigma}^{(1)} \cdot \mathbf{n}^{(1)}+$ $\boldsymbol{\sigma}^{(2)} \cdot \mathbf{n}^{(2)}$ and tensor $\boldsymbol{\delta}_{s} \equiv \boldsymbol{\delta}_{s}^{(1)} \cdot \boldsymbol{\tau}_{s}^{(2)}=\boldsymbol{\delta}_{s}^{(2)} \cdot \boldsymbol{\tau}_{s}^{(1)}$ have appeared consistently as in Truster and Masud (2014). The authors choose to neglect this term in the derivations that follow because it was found to have negligible contribution to numerical stability.

The variational multiscale interface framework has yielded stable and accurate results for linear elastostatics (Truster and Masud 2014), evolving finite deformations (Truster et al. 2015a), and plasticity (Truster 2015), particularly for nonconforming meshes and heterogeneous materials. Applying the framework to the transient problem provides consistent definitions for the interface flux terms according to Eq. (21). The performance of these fluxes at suppressing spurious oscillations arising at nonconforming interfaces under high-speed impact will be determined through numerical tests.

Remark: The appearance of the transient formulation in Eq. (24) is very much like the linear elastic formulation in Truster and Masud (2014). Indeed, the sole distinguishing features appear to be the presence of the inertial term $\mathbf{w} \cdot \rho / \beta \Delta t^{2} \mathbf{u}_{n+1}$ and the prescribed discontinuity $\zeta_{n+1}$. The implementation of the transient extension of the static formulation thus only requires the addition of these terms. However, in the preceding derivations, the fine-scale modeling assumptions that led to the simplified formulation in Eq. (24) have been carefully highlighted, namely that $\mathbf{u}^{\prime}$ is piecewise constant in time and localized to $\Gamma_{I}$. Thus, emphasis is placed on the treatment of interface dynamics; other fine-scale modeling techniques may provide improved performance in the bulk domain, such as for highly turbulent fluid dynamics (Calderer and Masud 2013).

\section{Extension to Transient Contact and Friction Problems}

Proceeding along the lines of Masud et al. (2012) and Truster et al. (2013) for the quasi-static case, the stabilized elastodynamic primal interface method is now extended to accommodate contact and friction mechanics. The key feature of contact problems (Masud et al. 2012; McDevitt and Laursen 2000; Simo and Laursen 1992) is that the interface continuity condition in Eq. (6) is replaced by an impenetrability condition in the normal direction and a stickslip relation in the tangential direction along $\Gamma_{I}$. Thus, the numerical flux terms contained in Eq. (24) is decomposed to treat each of these conditions separately, leading to the following semidiscrete formulation posed at time $t_{n+1}$ :

$$
\begin{aligned}
& \sum_{\alpha=1}^{2} \underbrace{\int_{\Omega^{(\alpha)}} \mathbf{w}^{(\alpha)} \cdot \frac{\rho^{(\alpha)}}{\beta \Delta t^{2}} \mathbf{u}_{n+1}^{(\alpha)} \mathrm{d} \Omega}_{\text {bulk inertia }}+\sum_{\alpha=1}^{2} \underbrace{\int_{\Omega^{(\alpha)}} \boldsymbol{\varepsilon}\left(\mathbf{w}^{(\alpha)}\right): \mathbf{C}: \boldsymbol{\varepsilon}\left(\mathbf{u}_{n+1}^{(\alpha)}\right) \mathrm{d} \Omega}_{\text {bulk stiffness }} \\
& -\underbrace{\int_{\Gamma_{I}} \delta g_{N} t_{N, n+1} \mathrm{~d} \Gamma}_{\text {pressure continuity }}-\underbrace{\int_{\Gamma_{I}} \delta t_{N} g_{N, n+1} \mathrm{~d} \Gamma}_{\text {impenetrability constraint }} \\
& -\underbrace{\int_{\Gamma_{I}} \delta \mathbf{g}_{T} \cdot \mathbf{t}_{T, n+1} \mathrm{~d} \Gamma}_{\text {frictional stick-slip relation }}-\underbrace{\int_{\Gamma_{I}} \delta \mathbf{t}_{T} \cdot \mathbf{g}_{T, n+1} \mathrm{~d} \Gamma}_{\text {full-stick condition }} \\
& =\sum_{\alpha=1}^{2} \int_{\Omega^{(\alpha)}} \mathbf{w}^{(\alpha)} \cdot\left(\mathbf{b}_{n+1}^{(\alpha)}-\rho^{(\alpha)} \tilde{\mathbf{a}}_{n+1}^{(\alpha)}\right) \mathrm{d} \Omega+\sum_{\alpha=1}^{2} \int_{\Gamma_{h}^{(\alpha)}} \mathbf{w}^{(\alpha)} \cdot \mathbf{h}_{n+1}^{(\alpha)} \mathrm{d} \Gamma
\end{aligned}
$$

The definitions for the normal and tangential components follow from term-by-term comparison between Eqs. (24) and (25) according to Masud et al. (2012). The normal gap $g_{N}$ and tangential gap $\mathbf{g}_{T}$ represent the amount of penetration or slip along the contact surface, respectively:

$$
g_{N}=-\llbracket \mathbf{X}+\mathbf{u} \rrbracket \cdot \mathbf{n}^{(1)}, \quad \mathbf{g}_{T}=-\left[\mathbf{I}-\mathbf{n}^{(1)} \otimes \mathbf{n}^{(1)}\right] \llbracket \mathbf{X}+\mathbf{u} \rrbracket
$$

where $\mathbf{X}=$ spatial coordinate of the surface $\Gamma_{I}$ in the undeformed configuration. The decomposition is taken with respect to the unit normal $\mathbf{n}^{(1)}$, and hence requires evaluation through a closest-point projection operation. This operation is described in detail within the appendix of Masud et al. (2012). The quantities $\delta g_{N}$ and $\delta \mathbf{g}_{T}$ are the variational gaps in the normal and tangential directions, respectively

$$
\delta g_{N}=-\llbracket \mathbf{w} \rrbracket \cdot \mathbf{n}^{(1)}, \quad \delta \mathbf{g}_{T}=-\left[\mathbf{I}-\mathbf{n}^{(1)} \otimes \mathbf{n}^{(1)}\right] \llbracket \mathbf{w} \rrbracket
$$

The contact pressure $t_{N}$ and shearing traction $\mathbf{t}_{T}$ are defined from the numerical flux terms in Eq. (21) in order to have equivalence with Eq. (24)

$$
\begin{gathered}
t_{N}=\left\{[\mathbf{C : \varepsilon}(\mathbf{u}) \cdot \mathbf{n}]-\boldsymbol{\tau}_{s} \llbracket \mathbf{X}+\mathbf{u} \rrbracket\right\} \cdot \mathbf{n}^{(1)} \\
\mathbf{t}_{T}=\left[\mathbf{I}-\mathbf{n}^{(1)} \otimes \mathbf{n}^{(1)}\right] \cdot\left\{[\mathbf{C}: \boldsymbol{\varepsilon}(\mathbf{u}) \cdot \mathbf{n}]-\boldsymbol{\tau}_{s} \llbracket \mathbf{X}+\mathbf{u} \rrbracket\right\}
\end{gathered}
$$

These expressions have an augmented form combining the traction field and displacement jump, similar to Simo and Laursen (1992). The variational tractions $\delta t_{N}$ and $\delta \mathbf{t}_{T}$ are defined analogously 


$$
\begin{gathered}
\delta t_{N}=\{\mathbf{C}: \boldsymbol{\varepsilon}(\mathbf{w}) \cdot \mathbf{n}\} \cdot \mathbf{n}^{(1)} \\
\delta \mathbf{t}_{T}=\left[\mathbf{I}-\mathbf{n}^{(1)} \otimes \mathbf{n}^{(1)}\right] \cdot\{\mathbf{C}: \boldsymbol{\varepsilon}(\mathbf{w}) \cdot \mathbf{n}\}
\end{gathered}
$$

Remark: The preceding definitions for the normal and tangential components are valid for small-deformation contact mechanics. Extension to large-deformation contact requires treatment of the variation of the unit normal vector; see, e.g., Wriggers et al. (1990) for discussions of this variation.

The tangential terms $\left\{\mathbf{g}_{T}, \delta \mathbf{g}_{T}\right\}$ and $\left\{\mathbf{t}_{T}, \delta \mathbf{t}_{T}\right\}$ provide a mechanism for embedding constitutive models for friction along the lines developed in Masud et al. (2012) and Truster et al. (2013). The variational traction term $\delta \mathbf{t}_{T}$ must be included when the constitutive response in the stick regime is desired to be completely rigid, similar to the augmented Lagrangian method (Simo and Laursen 1992) and the rigid-plastic frictional Nitsche formulation (Annavarapu et al. 2014). Herein, the elastoplastic regularized Coulomb friction model is employed as presented in Simo and Laursen (1992) and Truster et al. (2013) and the tangential traction $\mathbf{t}_{T}$ in Eq. (29) is replaced by the following evolution equations:

$$
\begin{gathered}
\Phi=\left\|\mathbf{t}_{T}\right\|+\mu_{f} t_{N} \leq 0, \quad \zeta \geq 0, \quad \zeta \Phi=0 \\
\dot{\mathbf{g}}_{T}-\zeta \frac{\partial}{\partial \mathbf{t}_{T}} \Phi=\frac{1}{\epsilon_{T}} \dot{\mathbf{t}}_{T}
\end{gathered}
$$

where $\mu_{f}=$ friction coefficient; and $\epsilon_{T}=$ tangential stiffness of the interface capturing the experimentally measured compliance (Wriggers et al. 1990). The elastic regularization in Eq. (33) provides an explicit evolution of $\mathbf{t}_{T}$ in terms of the increasing slip $\dot{\boldsymbol{g}}_{T}$. Therefore, the variational traction term $\delta \mathbf{t}_{T}$, which is generally variationally consistent only for rigid-plastic constitutive response, will be dropped from the formulation in Eq. (25).

The constitutive relations in Eqs. (32) and (33) are integrated in time using the backward Euler method and the radial return algorithm as described in Truster et al. (2013)

$$
\begin{gathered}
\mathbf{t}_{T, n+1}^{(\text {trial })}=\mathbf{t}_{T, n}+\epsilon_{T}\left(\mathbf{g}_{T, n+1}-\mathbf{g}_{T, n}\right) \\
\Phi_{n+1}^{(\text {trial })}=\left\|\mathbf{t}_{T, n+1}^{\text {(trial })}\right\|-\mu_{f} t_{N, n} \\
\mathbf{t}_{T, n+1}=\mathbf{t}_{T, n+1}^{\text {(trial })}-\Delta \zeta \frac{\mathbf{t}_{T, n+1}^{\text {(trial })}}{\left\|\mathbf{t}_{T, n+1}^{\text {(trial })}\right\|} \\
\Delta \zeta= \begin{cases}0 & \text { if } \Phi_{n+1}^{(\text {trial })} \leq 0 \\
\frac{\Phi_{n+1}^{(\text {trial })}}{\epsilon_{T}} & \text { if } \Phi_{n+1}^{(\text {trial })}>0\end{cases}
\end{gathered}
$$

Depending on whether the stick or slip regime is active, these expressions can be combined and simplified as follows:

$$
\begin{gathered}
\mathbf{t}_{T, n+1}=\mathbf{t}_{T, n}+\epsilon_{T}\left(\mathbf{g}_{T, n+1}-\mathbf{g}_{T, n}\right) \quad\left\|\mathbf{t}_{T, n+1}\right\| \leq \mu_{f} t_{N, n+1} \\
\mathbf{t}_{T, n+1}=\mu_{f} t_{N, n+1}\left(\mathbf{g}_{T, n+1}-\mathbf{g}_{T, n}\right) /\left\|\mathbf{g}_{T, n+1}-\mathbf{g}_{T, n}\right\| \\
\left\|\mathbf{t}_{T, n+1}\right\|>\mu_{f} t_{N, n+1} \quad(\text { slip })
\end{gathered}
$$

The backward Euler integration scheme for the constitutive model is consistent with the Newmark algorithm, which also evaluates momentum balance at $t_{n}$ and $t_{n+1}$. Substituting the constitutive model in Eqs. (26)-(39) into the contact weak form in Eq. (25) yields a nonlinear system of evolution equations that may be solved using the Newton-Raphson method. The linearization of the contact terms in Eq. (25) are contained in Masud et al. (2012) and references therein.

Remark: Presently, the two-body contact problem yields an inequality constraint expressed through a Kuhn-Tucker condition as $g_{N} \geq 0, t_{N} \leq 0$, and $g_{N} t_{N}=0$. Also, the tangential expressions in Eqs. (38) and (39) are valid only in the contact regime $t_{N}<0$. In the numerical setting, these conditions are evaluated at the Gauss points of the segments $\gamma_{s}$ along the interface. The traditional active set strategy (Simo and Laursen 1992) is employed by testing these conditions at each quadrature point and retaining interface quantities only for points in active contact.

Remark: The average acceleration method is utilized in the numerical problems herein for integrating the transient problem in Eq. (25), which is known to conserve energy in the linear context. However, this conservation is not guaranteed for nonlinear problems (Hughes 1976). Other techniques have been devised to improve the transient stability properties (Chung and Hulbert 1993; Love and Laursen 2003). Nonetheless, the authors are interested in investigating the stability of the response achieved by the consistently derived numerical fluxes in Eq. (21) within the standard Newmark algorithm. The present formulation could have been developed in the context of these enhanced time integration schemes.

Remark: Due to the stick-slip nature of the flow rule in Eq. (33), the consistent tangent matrix in regions with persistent sliding is indeterminate between the elastic and plastic branches for the first iteration of a load step. The numerical studies herein indicate that using the plastic or slip constitutive tangent as the predictor enhanced the stability of the method, enabling larger time steps. Similar observations were made in the context of cohesive-zone modeling for delamination (Alfano and Crisfield 2001).

\section{Generalization to Other One-Step Integration Methods}

Upon applying spatial discretization, the stabilized interface weak form presented in Eq. (25) leads to the discrete momentum balance equation that can be represented in vector form as

$$
\mathbf{M} \mathbf{a}_{n+1}+\mathbf{K}^{\text {bulk }} \mathbf{d}_{n+1}+\mathbf{F}^{\text {contact }}\left(\mathbf{d}_{n+1}\right)=\mathbf{F}^{\text {external }}\left(t_{n+1}\right)
$$

where $\mathbf{M}=$ mass matrix; $\mathbf{K}^{\text {bulk }}=$ stiffness contribution of the interior of domain $\Omega ; \mathbf{F}^{\text {external }}\left(t_{n+1}\right)=$ external force vector; and $\mathbf{F}^{\text {contact }}\left(\mathbf{d}_{n+1}\right)=$ nonlinear term arising from contact and friction along $\Gamma_{I}$. Although the development of the semidiscrete stabilized form has been presented within the context of the standard Newmark method, the authors wish to emphasize that the proposed method can be easily cast in any other time integrator of choice. A commonly used method is the generalized- $\alpha$ method (Chung and Hulbert 1993) that provides targeted damping in the higher modes while maintaining second-order accuracy and minimizing damping in the lower modes. These features make this algorithm attractive for structural dynamics problems. In extending the proposed method to the generalized- $\alpha$ format, the predictor and multicorrector Eqs. (8) and (9) do not change, only the time discrete equation of motion in Eq. (40) gets updated as follows:

$\mathbf{M a}_{n+1-\alpha_{m}}+\mathbf{K}^{\text {bulk }} \mathbf{d}_{n+1-\alpha_{f}}+\mathbf{F}^{\text {contact }}\left(\mathbf{d}_{n+1-\alpha_{f}}\right)=\mathbf{F}^{\text {external }}\left(t_{n+1-\alpha_{f}}\right)$ 


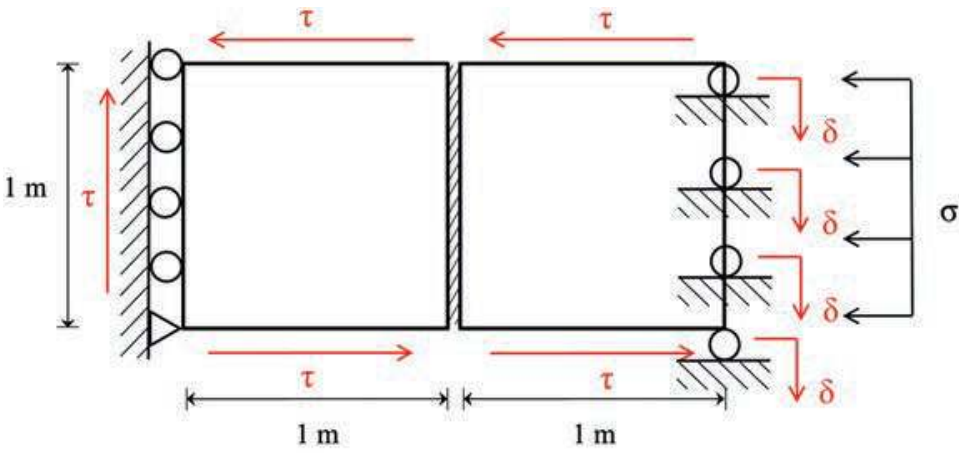

(a)

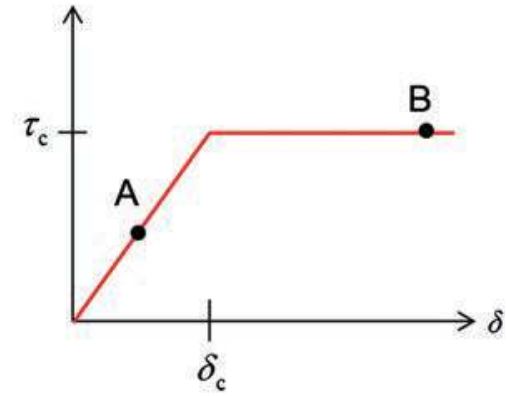

(b)

Fig. 2. (Color) Contact patch test: (a) domain and boundary conditions; (b) variation of applied load

$$
\begin{aligned}
\mathbf{d}_{n+1-\alpha_{f}} & =\left(1-\alpha_{f}\right) \mathbf{d}_{n+1}+\alpha_{f} \mathbf{d}_{n} \\
\mathbf{a}_{n+1-\alpha_{m}} & =\left(1-\alpha_{m}\right) \mathbf{a}_{n+1}+\alpha_{m} \mathbf{a}_{n} \\
t_{n+1-\alpha_{f}} & =\left(1-\alpha_{f}\right) t_{n+1}+\alpha_{f} t_{n}
\end{aligned}
$$

Within the nonlinear momentum balance in Eq. (41), the contact terms defined in the previous section that are contained in $\mathbf{F}^{\text {contact }}$ are evaluated at the intermediate time $t_{n+1-\alpha_{f}}$. Consistent linearization of Eq. (41) leads to a consistent tangent matrix that has the same structure as that of the Newmark method, except that it contains additional factors of $\left(1-\alpha_{f}\right)$ and $\left(1-\alpha_{m}\right)$. All other developments remain essentially unaltered. This direct extension is enabled by the piecewise constant in time approximation of the fine scales.

\section{Numerical Results}

The performance of the proposed dynamic interface method is assessed through a series of numerical benchmark problems. Emphasis is placed on the effects that mesh conformity and friction have on the computed response. Plane strain conditions are assumed in all cases. Bilinear quadrilateral elements are employed, and full numerical quadrature is used to evaluate the domain and interface integrals. Details concerning the integration of the interface terms and the definitions of the bubble functions used within the stability tensors in Eq. (18) are found in Masud et al. (2012), Truster et al. (2015b), and Truster and Masud (2014). For the frictional problem, the Newton-Raphson algorithm with consistent linearization is employed to solve Eq. (25) at each time step, and quadratic rate of convergence of the out-of-balance residual vector is achieved.

\section{Quasi-Static Patch Test for Frictional Response}

To verify the consistency of the method, a quasi-static friction problem with an exact solution is investigated. Consider two blocks joined by a frictional interface that are pressed together by a constant applied pressure $\sigma$ as shown in Fig. 2. Shearing tractions $\tau$ are applied on all faces to create a uniform state of stress, except for the right face where a uniform prescribed displacement $\delta$ is applied to each of the rollers in the vertical direction. The displacement and the tractions are increased proportionally up to the critical stick-slip transition at $\delta_{c}$ and $\tau_{c}$. Afterward, the displacement can be increased to freely slide the surfaces of the blocks past each other. On the finite-element mesh, the time-varying vertical displacement $\delta$ is prescribed to each of the nodes along the right face of the domain.

For the numerical simulation, each block is discretized into a nonuniform mesh of four quadrilateral elements as shown in Fig. 3. The material properties are taken as $G=5 \mathrm{kPa}, \nu=0$, $\epsilon_{T}=100 \mathrm{kN} / \mathrm{m}$, and $\mu_{f}=0.25$. The compressive stress is set to $\sigma=40 \mathrm{~Pa}$ such that the critical shear stress to induce sliding is $\tau_{c}=\mu_{f} \sigma=10 \mathrm{~Pa}$; the axial compression does not induce lateral expansion because $\nu=0$. Due to the tangential elasticity of the interface, the deflection of the right block at the critical point is determined as $\delta_{c}=\tau_{c} L / G+\tau_{c} / \epsilon_{T}=0.0041 \mathrm{~m}$, where $L=$ $2 \mathrm{~m}$ is the length of the two blocks. In the numerical test, the shear stress is first increased in two steps using $\Delta \tau=5 \mathrm{~Pa}$ and displacement $\Delta \delta=0.00205 \mathrm{~m}$; the end of the first step is denoted by A in Fig. 2(b). This applied displacement increment is then maintained after the critical slip state for four more steps to a maximum value of $\delta=0.0123 \mathrm{~m}$, which is indicated by point $\mathrm{B}$. Because the total slip is less than $1 \%$ of the domain width, the problem has been simulated with the normal vector and contact surfaces evaluated in the undeformed configuration in order to accommodate an analytical solution. The stress contours at these two states are shown in

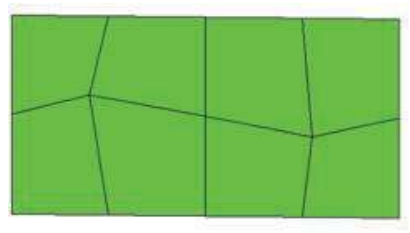

(a)
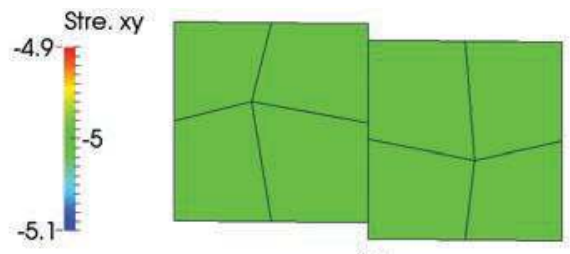

(b)

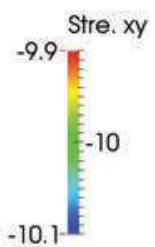

Fig. 3. (Color) Stress contours $\tau_{x y}$ on amplified deformed configuration: (a) applied displacement $\delta=0.00205 \mathrm{~m}$; (b) applied displacement $\delta=0.01230 \mathrm{~m}$ 


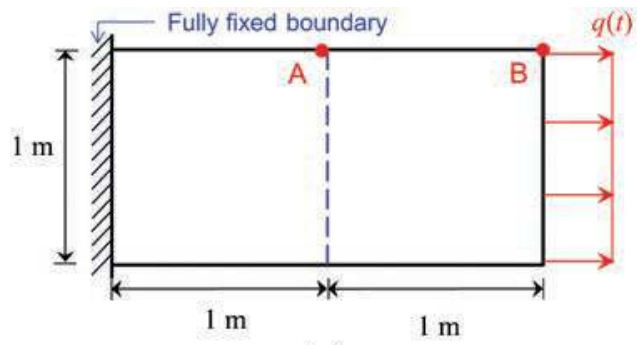

(a)

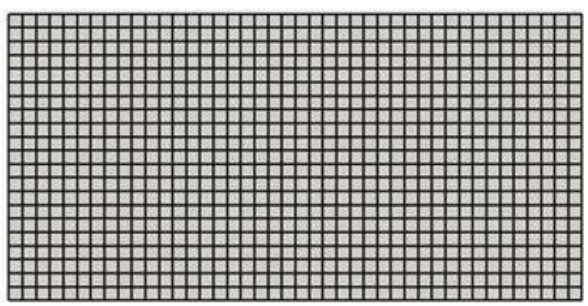

(b)

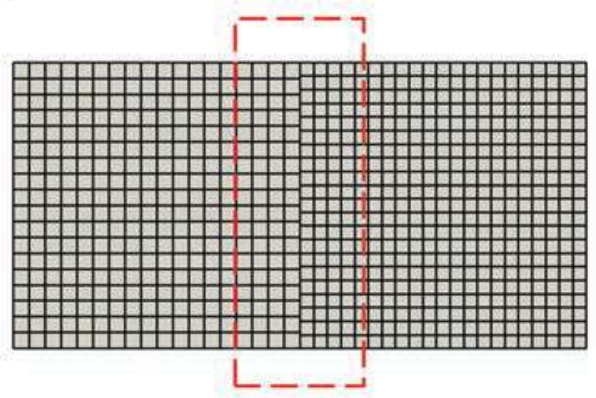

(c)

Fig. 4. (Color) Problem description: (a) axial bar domain: (b) conforming mesh; (c) nonconforming mesh

Fig. 3. The deformations of the domains have been amplified by a factor of 10 to highlight the discontinuity after the critical stress is achieved in Fig. 3(b). Clearly, the computed stresses are constant within the two blocks as is expected from the exact solution. Thus, the stabilized DG interface method enforces the impenetrability constraint exactly and reproduces the various stages of stick and slip.

\section{Axial Bar under Tensile Impact}

The next numerical test investigates whether the insertion of a discrete interface into an otherwise continuous domain affects the underlying dynamic response. The domain consists of a rectangular bar that is fully fixed on the left edge, as shown in Fig. 4(a), and a Heaviside tensile traction is applied horizontally on the right edge with a magnitude of $q_{o}=1 \mathrm{MPa}$. The material properties are taken as $E=210 \mathrm{GPa}, \nu=0.3, \rho=7,800 \mathrm{~kg} / \mathrm{m}^{3}$, and $t=1 \mathrm{~m}$ in order to agree with a study conducted by Herry et al. (2002), which utilized Lagrange multipliers to enforce the interface constraints. The average acceleration method $(\beta=1 / 4, \gamma=1 / 2)$ was used for time integration with a time step of $\Delta t=12.5 \mu \mathrm{s}$. A series of four analyses are performed. A conforming continuous Galerkin (CG) mesh with $21 \times 42$ elements serves as a benchmark wherein an interface is not inserted, as shown in Fig. 4(b). The second analysis uses the same mesh, but a vertical DG interface is applied in the center that partitions the domain into two squares of $21 \times 21$ elements. The other two analyses are conducted on the mesh in Fig. 4(c) containing a nonconforming DG interface. The interface, which is highlighted by the red dashed box around the center of the mesh, arises because different numbers of elements are used in the two regions $(18 \times 18$ in the left half, $21 \times 21$ in the right half $)$. As mentioned in Herry et al. (2002), this mesh does not contain any element edges that match up along the interface. The first simulation on this mesh will employ the definitions of the stability tensor and flux weights defined in Eqs. (18) and (23), and the results will be labeled as VMDG according to the name given in Truster et al. (2015b). Second, the results produced by the classical parameters from the DG method (Arnold et al. 2002) are also investigated. Thus, equal scalar weights $\delta_{s}^{(1)}=\delta_{s}^{(2)}=1 / 2$ are applied in the interface flux, and the area-weighted formula for the stability parameter is taken from Masud et al. (2012)

$$
\tau_{s}=\frac{5\left[G^{(1)}+G^{(2)}\right]}{h_{s}}, \quad h_{s}=2\left\{\frac{\operatorname{meas}\left[\Gamma_{e}^{(1)}\right]}{\operatorname{meas}\left[\Omega_{e}^{(1)}\right]}+\frac{\operatorname{meas}\left[\Gamma_{e}^{(2)}\right]}{\operatorname{meas}\left[\Omega_{e}^{(2)}\right]}\right\}^{-1}
$$

where $\Omega_{e}^{(1)}$ and $\Omega_{e}^{(2)}=$ elements on either side of the segment $\gamma_{s}$; and $\Gamma_{e}^{(\alpha)}=$ entire edge of the element $\Omega_{e}^{(\alpha)}$. These results will be labeled as equal discontinuous Galerkin (eDG). In contrast, the tensors $\boldsymbol{\tau}_{s}^{(\alpha)}$ are evaluated over sectors $\omega_{s}^{(\alpha)}$ of the interface, which are defined according to the automatic procedure in Truster and Masud (2014) and have variable size due to the nonconformity of $\Gamma_{I}$. Again, the intentions for this problem are to study the effect of the definition for the stability parameters in the method and to determine if the discrete interface introduces undesirable numerical artifacts.

Simulations are performed on each mesh to track the dominant longitudinal vibrations induced by the Heaviside force; minor transverse modes are also excited due to the Poisson effect and the fully fixed left edge. A contour plot of the axial stress $\sigma_{x x}$ obtained from each mesh during the first cycle at time $t=750 \mu \mathrm{s}$ when the bar is beginning to recoil is shown in Figs. 5(a-d), where the deformations are magnified 10,000 times. The stress field is nonuniform due to the higher-frequency dynamical effects induced from the boundary conditions, and a finer grid may be required to fully resolve these details. However, Figs. 5(a and b) clearly indicate that the conforming DG mesh produces essentially the same response as the benchmark CG mesh. Also, the two nonconforming simulations have produced nearly identical stress contours in Figs. 5(c and d). While the conforming and nonconforming results appear qualitatively similar, a close inspection indicates that the axial stress wave has travelled slightly further to the right on the nonconforming mesh. This apparent difference in the wave speed from the stress contour plots is likely caused by the different sizes of the solid elements in the two meshes rather than being caused by the DG interface. Also recall that the accuracy of the stress field is one order less than the displacement field for finite-element 


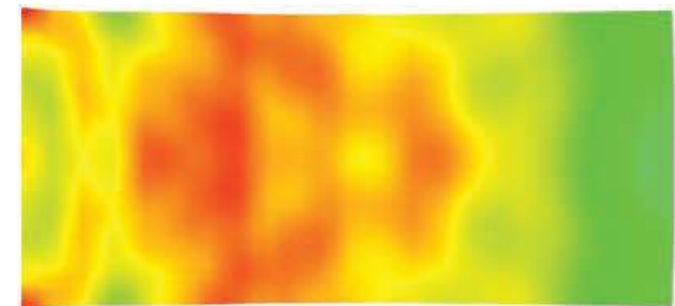

(a)

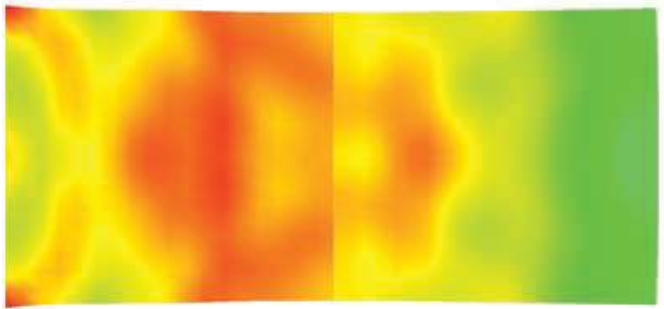

(c)
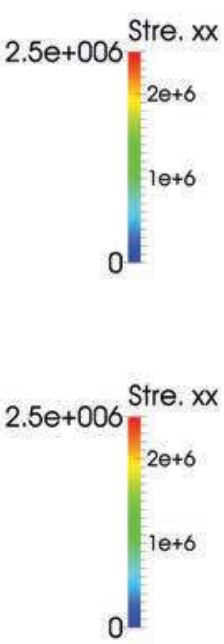
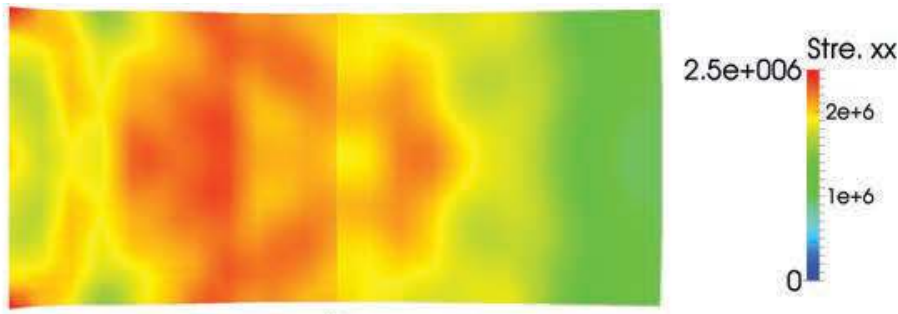

(b)
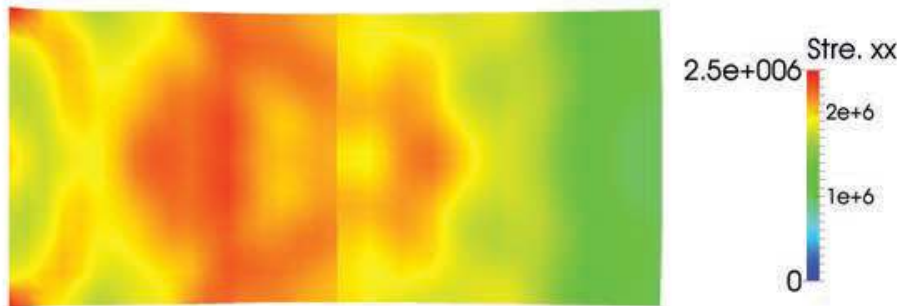

(d)

Fig. 5. (Color) Stress $\sigma_{x x}$ (Pa) contour plots of response at time $t=750 \mu \mathrm{s}$ : (a) CG mesh; (b) VMDG conforming mesh; (c) VMDG nonconforming mesh; (d) eDG nonconforming mesh
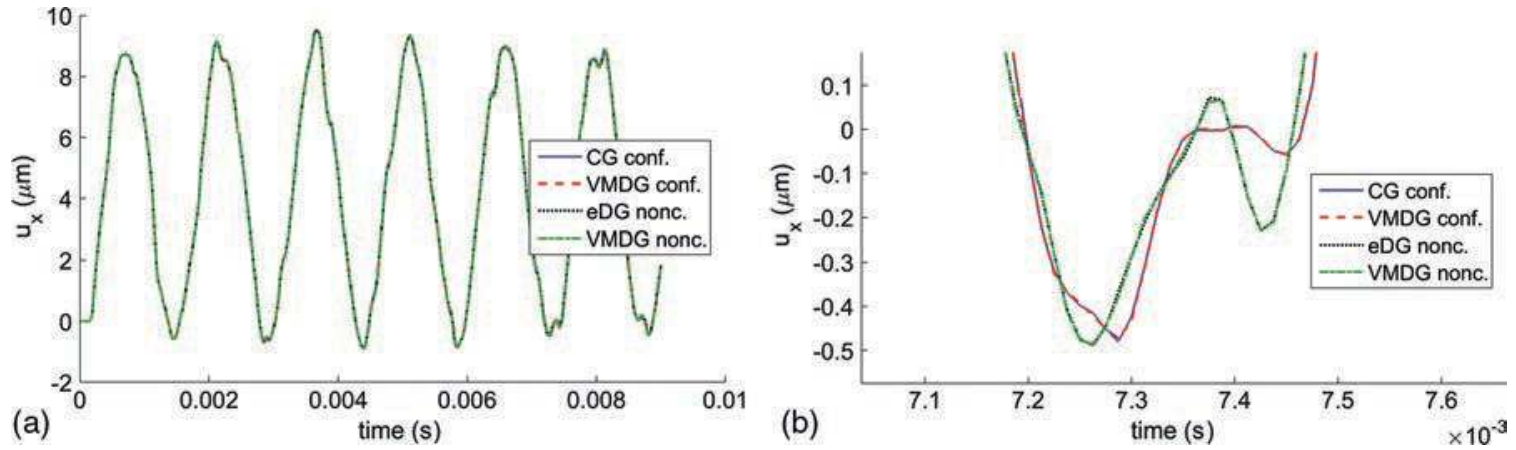

Fig. 6. (Color) Displacement $u_{x}(\mu \mathrm{m})$ at Point A: (a) six cycles; (b) focus on $7.3 \mathrm{~ms}$

models, and the displacement accuracy is compared in the subsequent figures. Thus, the interface $\Gamma_{I}$ does not appear to induce artificial dispersion or scattering, at least not during the first cycle of transient response.

Next, the time history of the axial displacement $u_{x}$ obtained at a point on the interface [Point A in Fig. 4(a)] is depicted in Fig. 6 for each of the analyses. All four curves appear to lie exactly on top of each other during the six cycles of response shown in Fig. 6(a). By zooming in at the instant $7.3 \mathrm{~ms}$, the responses from the conforming meshes and the nonconforming meshes can be distinguished. Even with this magnification, the CG and VMDG conforming results exhibit complete agreement. Slight differences can be seen between the VMDG and eDG results for the nonconforming mesh. The nonconforming mesh exhibits slightly higher frequency response than the conforming mesh, which is logical due to the additional degrees of freedom at the interface. Also, the displacement history results agree very closely with those reported in Herry et al. (2002); note that the time step size was $\Delta t=0.125 \mu \mathrm{s}$ in the reference. Thus, the differences in the transient response induced by the definition of the stability parameters at the interface appear to be rather mild for this benchmark problem. Nonetheless, the authors with to highlight that this optimal performance was achieved by the VMDG method for the nonconforming mesh without requiring any user calibration of the interface stabilizing terms. The consistently derived expressions in Eqs. (18) and (23) provided a proper accounting for the difference in element size and edge mismatch along $\Gamma_{I}$.

Remark: During prolonged simulations for 200 cycles, neither phase error nor amplitude error developed in the primary axial mode between the conforming meshes and nonconforming. This result indicates that artificial diffusion is not introduced by the interface.

Analogous plots are provided for the velocity $v_{x}$ at Point $\mathrm{A}$ for each mesh in Fig. 7. The differences in the four simulations are more clearly observed in the magnified plot in Fig. 7(b); even the CG and DG results on the conforming mesh have slight variations. The rather jagged response is attributed to the fixed boundary conditions on the left end of the bar, particularly because the initial response of the bar before $0.25 \mathrm{~ms}$ appears to be smooth.

As a quantitative indicator of the differences in the solutions, a record of the nodal displacement value at Point B [Fig. 4(a)] on the right end of the bar obtained from each analysis is provided in Table 1 . The slightly smaller displacements for the nonconforming mesh indicate that the recoil of the first cycle has progressed further than the conforming mesh, which agrees with the stress contours shown in Fig. 5.

Remark: Observe that the expressions for the interfacial stability parameters $\boldsymbol{\tau}_{s}$ and $\boldsymbol{\delta}_{s}^{(\alpha)}$ in Eq. (23) informed by the fine-scale 

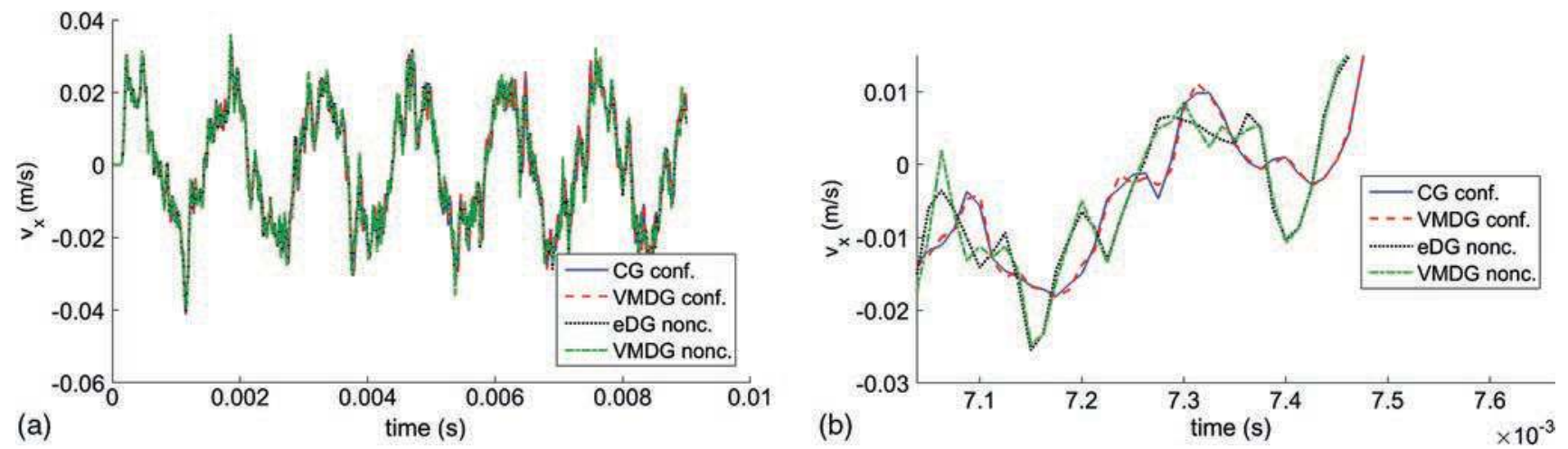

Fig. 7. (Color) Velocity $v_{x}(\mathrm{~m} / \mathrm{s})$ at Point A: (a) six cycles; (b) focus on $7.3 \mathrm{~ms}$

Table 1. Displacement $\mathbf{u}_{x}$ of Point B at Time $t=750 \mu \mathrm{s}$

\begin{tabular}{lc}
\hline Mesh & Value $(\mu \mathrm{m})$ \\
\hline CG conforming & 15.57442 \\
VMDG conforming & 15.57417 \\
VMDG nonconforming & 15.56408 \\
eDG nonconforming & 15.56557 \\
\hline
\end{tabular}

models in Eq. (18) account for element size disparity across the interface $\Gamma_{I}$. This fact enables the VMDG method to achieve accurate and nondispersive results for the nonconforming mesh.

\section{Bolted Lap Joint under Tensile Loading}

The final problem is of physical significance: a bolted lap joint loaded by a tensile impact force. The joint consists of two 1-cm-thick, $25-\mathrm{cm}$-long plates attached by $12-\mathrm{mm}$-diameter bolts to lap plates that are $0.5 \mathrm{~cm}$ thick and $12 \mathrm{~cm}$ long, as shown in Fig. 8(a). These dimensions are representative of an experimental test conducted in Eriten et al. (2011). To approximate the bolts within a two-dimensional model, a uniform pressure $P=35 \mathrm{MPa}$ is applied over 20 -mm-wide zones as indicated in the figure. This pressure corresponds to individual bolts at a $5-\mathrm{cm}$ lateral spacing with a $35-\mathrm{kN}$ pretension, as illustrated in Fig. 8(b). The material parameters for steel are taken as $E=200 \mathrm{GPa}, \nu=0.24$, and $\rho=7,800 \mathrm{~kg} / \mathrm{m}^{3}$, and the frictional parameters are taken as
$\varepsilon_{T}=5 \times 10^{4} \mathrm{GN} / \mathrm{m}$ and $\mu_{f}=0.30$. The joint is simulated using the trapezoidal rule and a time step $\Delta t=6.25 \mu \mathrm{s}$, and the magnitude of the tensile impact traction (applied instantly at $t=0$ and held constant) is $F_{0}=5 \mathrm{MPa}$, applied uniformly along the right edge. For the discretization, a conforming mesh is used with three elements through the thickness of the lap plates, shown in Fig. 8(c). The influence of mesh refinement for problems with friction has been treated for the quasi-static case in Truster et al. (2013). A fully fixed boundary condition is applied on the left edge.

A major quantity of interest is the evolution of the axial stress waves induced by the application of the tensile force. While the joint induces additional effects, the dominant response is a longitudinal vibration and associated stress wave similar to the previous problem. A snapshot of the stress contour is provided in Fig. 9 during the first cycle when the wave is reflecting off the fixed end, where the overall joint response is given in Fig. 9(a). Three separate interface conditions are compared, which are shown zoomed in over the center of the joint. In Figs. 9(c and d), the plates are welded together along $60 \%$ of each of the contacting interfaces, nearly matching the persistent contact zone caused by the bolts. For the configuration shown in Fig. 9(c), the nodal values are tied together to give a CG discretization, while full DG treatment coupling normal and tangential interactions is utilized in Fig. 9(d); hence the surfaces are welded together. These configurations serve as a reference for analyzing the dynamics of the contact and Coulomb friction response in Fig. 9(b). In all three cases, the bolt preload causes the toes of the lap plates to rise off of

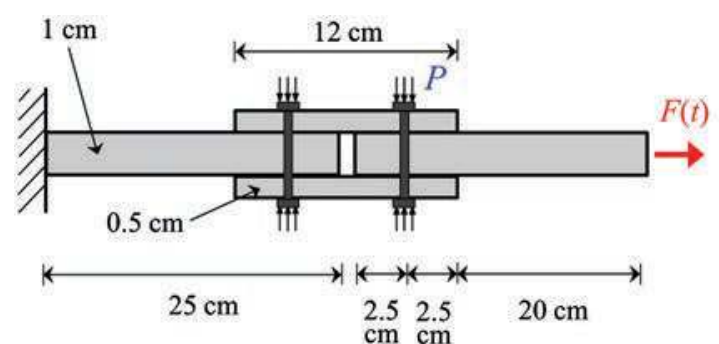

(a)

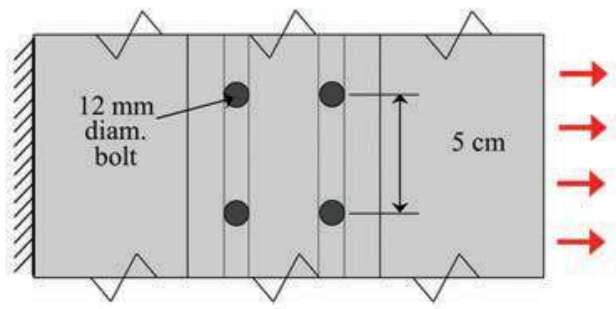

(b)

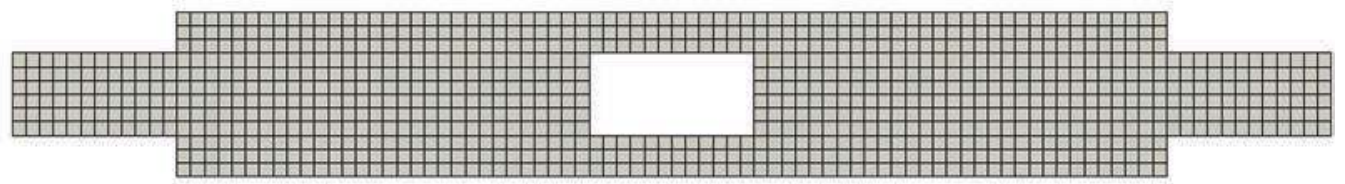

(c)

Fig. 8. (Color) Double-bolted lap joint: (a) side view; (b) plan view; (c) conforming discretization 
Stre. $X X$

$-4.0 e+006 \quad 0.0 e+000 \quad 4.0 e+006 \quad 8.0 e+006 \quad 1.2 e+007$

$-6.7 e+006 \quad 1.5 e+007$

(a)

CG Welded Config

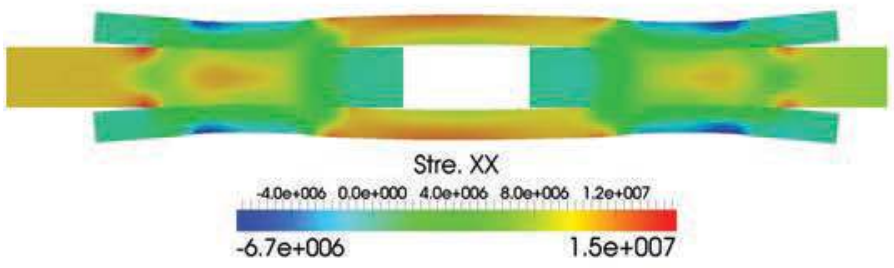

(c)

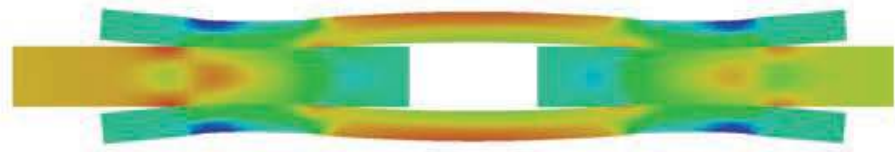

Stre. $X X$

$4.00+006 \quad 0.00+000 \quad 4.00+006 \quad 8.00+006 \quad 1.20+007$

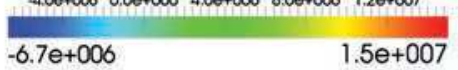

(b)

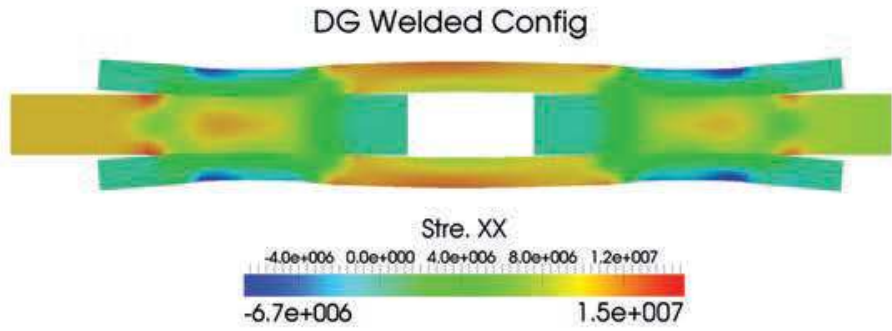

(d)

Fig. 9. (Color) Axial stress contour at time $t=0.00025 \mathrm{~s}$ : (a) overview of entire joint; (b) bolted simulation; (c) welded contact using nodal tying; (d) welded contact using DG method

the long plates. The contours of the two welded configurations in Figs. 9(c and d) are identical; therefore, all variations between the welded and bolted configurations can be attributed to the dynamics of contact and friction. In particular, note that the stress wave appears to have progressed slightly further in the bolted configuration. Also, the stress in the contacting zone appears a bit different near the outer edges.

To further highlight the features of the dynamic response, an additional set of contour plots corresponding to the end of the cycle are shown in Fig. 10. Compression is evident in the lap plates at this instant in time, and the profiles from the bolted and welded configurations are again somewhat distinct.

\section{Axial Stress (CG)}

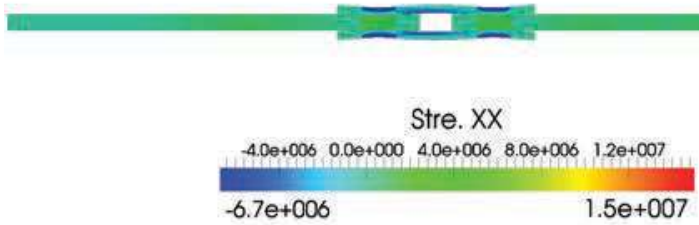

(a)

CG Welded Config.

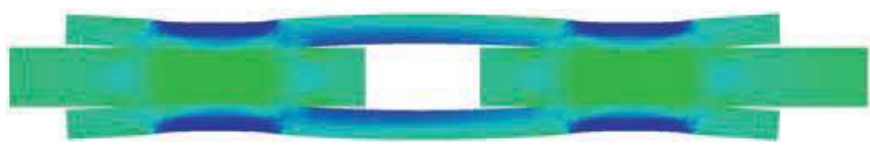

Stre. XX

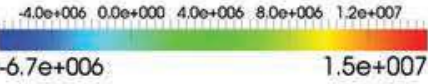

(c)
As a final result, the long-term behavior of the tip displacement is presented in Fig. 11. Because the two welded configurations had identical results, only the CG results are presented for clarity. Even after a few cycles, the frictional response is seen to deviate from the welded response. The primary mode is a see-sawing motion characteristic of a long one-dimensional rod, with high-frequency response likely induced by reflections in the lap plates. The simulation was carried out for nearly a tenth of a second, over which hundreds of cycles occur. From the displacement plot in Fig. 11(b), dissipation is evident in the bolted configuration while the welded configuration maintains constant system energy. Also, both configurations exhibit a beating phenomenon, although the frequency

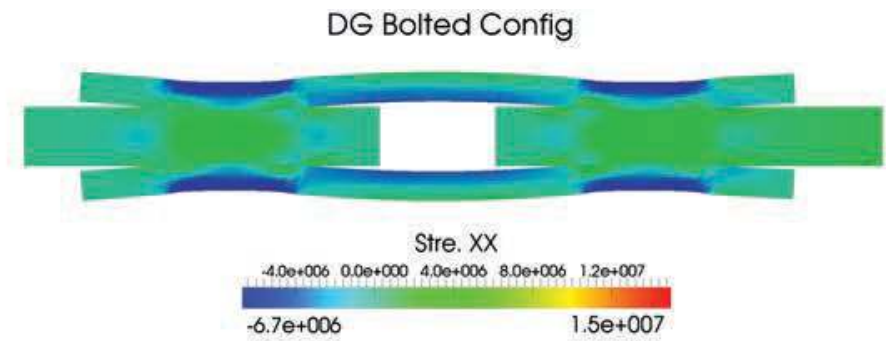

(b)

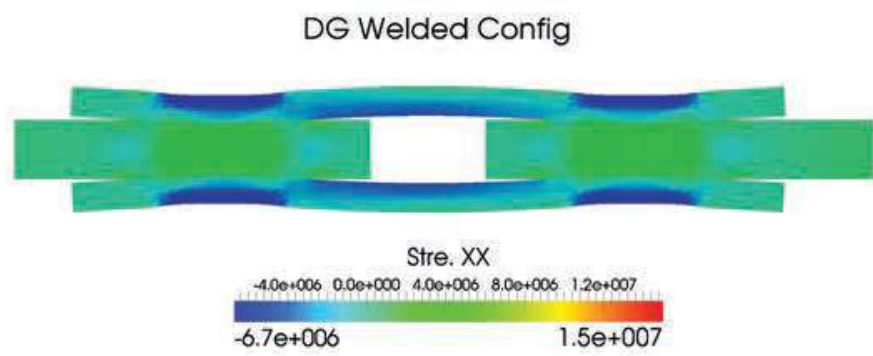

(d)

Fig. 10. (Color) Axial stress contour at time $t=0.000431 \mathrm{~s}$ : (a) overview of entire joint; (b) bolted simulation; (c) welded contact using nodal tying; (d) welded contact using DG method 

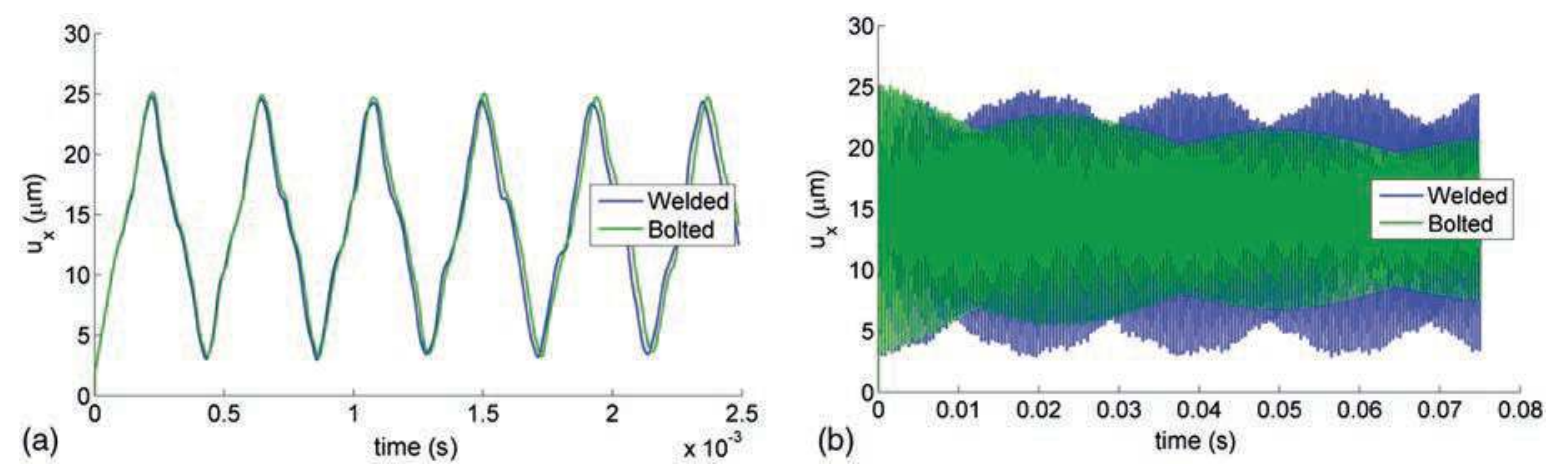

Fig. 11. (Color) Time history of horizontal tip deflection of lap joint: (a) displacement $u_{x}$ during six cycles; (b) long-term history of displacement $u_{x}$

of the frictional case is lower. These results help isolate the frictional effects on the underlying dynamics and demonstrate the performance of the method for solving interface problems.

\section{Conclusions}

A DG computational technique for modeling the transient response of domains containing interfaces is consistently derived by extending ideas from the stabilized primal interface framework of Truster and Masud (2014) to include time-dependent effects. Subsequently, the method is applied to frictional contact problems by embedding constitutive models for friction into the tangential numerical flux terms at the interface in lieu of Masud et al. (2012). A key benefit of the present approach is that the need for auxiliary Lagrange multipliers to treat the contact constraint is eliminated, reducing the cost of the method and avoiding the stability issues associated with mixed methods. Multiple nonsmooth transient problems were analyzed, including simulations on nonconforming meshes. The proposed DG method produces almost identical results to the classical CG method for the case of conforming meshes. Computed solution discrepancies observed for nonconforming interfaces were restricted to high-frequency modes. Total energy is conserved by the DG method for frictionless fully bonded interfaces when the average acceleration method is employed for time integration. Dissipation effects and beating phenomena are highlighted within a simulation of a bolted lap joint under impact loading.

\section{Acknowledgments}

This work was sponsored by National Science Foundation (NSF) Division of Civil, Mechanical and Manufacturing Innovation (CMMI) Grant No. 08-0020. This support is gratefully acknowledged.

\section{References}

Alfano, G., and Crisfield, M. A. (2001). "Finite element interface models for the delamination analysis of laminated composites: Mechanical and computational issues." Int. J. Numer. Methods Eng., 50(7), 1701-1736.

Anciaux, G., and Molinari, J. F. (2010). "Sliding of rough surfaces and energy dissipation with a 3D multiscale approach." Int. J. Numer. Methods Eng., 83(8-9), 1255-1271.

Annavarapu, C., Hautefeuille, M., and Dolbow, J. E. (2012a). "A robust Nitsches formulation for interface problems." Comput. Methods Appl. Mech. Eng., 225, 44-54.

Annavarapu, C., Hautefeuille, M., and Dolbow, J. E. (2012b). "Stable imposition of stiff constraints in explicit dynamics for embedded finite element methods." Int. J. Numer. Methods Eng., 92(2), 206-228.
Annavarapu, C., Hautefeuille, M., and Dolbow, J. E. (2014). "A Nitsche stabilized finite element method for frictional sliding on embedded interfaces. Part I: Single interface." Comput. Methods Appl. Mech. Eng., 268, 417-436.

Arnold, D. N., Brezzi, F., Cockburn, B., and Marini, L. D. (2002). "Unified analysis of discontinuous Galerkin methods for elliptic problems." SIAM J. Numer. Anal., 39(5), 1749-1779.

Barbosa, H. J. C., and Hughes, T. J. R. (1991). "The finite element method with Lagrange multipliers on the boundary: Circumventing the Babuška-Brezzi condition." Comput. Methods Appl. Mech. Eng., 85(1), 109-128.

Calderer, R., and Masud, A. (2013). "Residual-based variational multiscale turbulence models for unstructured tetrahedral meshes." Comput. Methods Appl. Mech. Eng., 254, 238-253.

Cervera, M., Chiumenti, M., Valverde, Q., and Agelet de Saracibar, C. (2003). "Mixed linear/linear simplicial elements for incompressible elasticity and plasticity." Comput. Methods Appl. Mech. Eng., 192(49-50), 5249-5263.

Chung, J., and Hulbert, G. M. (1993). "A time integration algorithm for structural dynamics with improved numerical dissipation: The generalized- $\alpha$ method." J. Appl. Mech., 60(2), 371-375.

Eriten, M., Polycarpou, A. A., and Bergman, L. A. (2011). "Physics-based modeling for fretting behavior of nominally flat rough surfaces." Int. $J$. Solids Struct., 48(10), 1436-1450.

Herry, B., Di Valentin, L., and Combescure, A. (2002). "An approach to the connection between subdomains with non-matching meshes for transient mechanical analysis." Int. J. Numer. Methods Eng., 55(8), 973-1003.

Hughes, T. J. R. (1976). "Stability, convergence and growth and decay of energy of the average acceleration method in nonlinear structural dynamics." Comput. Struct., 6(4-5), 313-324.

Hughes, T. J. R. (1995). "Multiscale phenomena: Green's functions, the Dirichlet-to-Neumann formulation, subgrid scale models, bubbles and the origins of stabilized methods." Comput. Methods Appl. Mech. Eng., 127(1-4), 387-401.

Ladeveze, P., Nouy, A., and Loiseau, O. (2002). "A multiscale computational approach for contact problems." Comput. Methods Appl. Mech. Eng., 191(43), 4869-4891.

Laursen, T. A., Puso, M. A., and Sanders, J. (2012). "Mortar contact formulations for deformable-deformable contact: Past contributions and new extensions for enriched and embedded interface formulations." Comput. Methods Appl. Mech. Eng., 205, 3-15.

Love, G. R., and Laursen, T. A. (2003). "Improved implicit integrators for transient impact problems-dynamic frictional dissipation within an admissible conserving framework." Comput. Methods Appl. Mech. Eng., 192(19), 2223-2248.

Masud, A., and Truster, T. J. (2013). "A framework for residual-based stabilization of incompressible finite elasticity: Stabilized formulations and methods for linear triangles and tetrahedra." Comput. Methods Appl. Mech. Eng., 267, 359-399.

Masud, A., Truster, T. J., and Bergman, L. A. (2011). "A variational multiscale a posteriori error estimation method for mixed form of nearly 
incompressible elasticity." Comput. Methods Appl. Mech. Eng., 200(47-48), 3453-3481.

Masud, A., Truster, T. J., and Bergman, L. A. (2012). "A unified formulation for interface coupling and frictional contact modeling with embedded error estimation." Int. J. Numer. Methods Eng., 92(2), 141-177.

Masud, A., and Xia, K. (2006). "A variational multiscale method for inelasticity: Application to superelasticity in shape memory alloys." Comput. Methods Appl. Mech. Eng., 195(33-36), 4512-4531.

McDevitt, T. W., and Laursen, T. A. (2000). "A mortar-finite element formulation for frictional contact problems." Int. J. Numer. Methods Eng., 48(10), 1525-1547.

Popp, A., and Wall, W. A. (2014). "Dual mortar methods for computational contact mechanics-Overview and recent developments." GAMMMitteilungen, 37(1), 66-84.

Scovazzi, G., Carnes, B., Zeng, X., and Rossi, S. (2015). "A simple, stable, and accurate tetrahedral finite element for transient, nearly and fully incompressible solid dynamics: A dynamic variational multiscale approach.” Int. J. Numer. Methods Eng., 106(10), 799-839.

Simo, J. C., and Laursen, T. A. (1992). "An augmented Lagrangian treatment of contact problems involving friction." Comput. Struct., 42(1), 97-116.

Sitzmann, S., Willner, K., and Wohlmuth, B. I. (2015). "A dual Lagrange method for contact problems with regularized frictional contact conditions: Modelling micro slip." Comput. Methods Appl. Mech. Eng., 285, 468-487.
Temizer, I. (2013). "A mixed formulation of mortar-based contact with friction." Comput. Methods Appl. Mech. Eng., 255, 183-195.

Truster, T. J. (2015). "A stabilized, symmetric Nitsche method for spatially localized plasticity." Comput Mech., 57(1), 75-103.

Truster, T. J., Chen, P., and Masud, A. (2015a). "Finite strain primal interface formulation with consistently evolving stabilization." Int. J. $\mathrm{Nu}$ mer. Methods Eng., 102(3-4), 278-315.

Truster, T. J., Chen, P., and Masud, A. (2015b). "On the algorithmic and implementational aspects of a discontinuous Galerkin method at finite strains." Comput. Math. Appl., 70(6), 1266-1289.

Truster, T. J., Eriten, M., Polycarpou, A. A., Bergman, L. A., and Masud, A. (2013). "Stabilized interface methods for mechanical joints: Physicsbased models and variationally consistent embedding." Int. J. Solids Struct., 50(14-15), 2132-2150.

Truster, T. J., and Masud, A. (2013). "A discontinuous/continuous Galerkin method for modeling of interphase damage in fibrous composite systems." Comput. Mech., 52(3), 499-514.

Truster, T. J., and Masud, A. (2014). "Primal interface formulation for coupling multiple PDEs: A consistent derivation via the variational multiscale method." Comput. Methods Appl. Mech. Eng., 268, 194-224.

Wriggers, P., Vu Van, T., and Stein, E. (1990). "Finite element formulation of large deformation impact-contact problems with friction." Comput. Struct., 37(3), 319-331.

Wriggers, P., and Zavarise, G. (2007). "A formulation for frictionless contact problems using a weak form introduced by Nitsche." Comput. Mech., 41(3), 407-420. 